\title{
Hábitos podológicos en personas con alteraciones ungueales
}

\author{
Abián Mosquera-Fernández1, María Díaz-Rodríguez², Cristina González-Martín, Marta Platero-Arboiro², \\ Victoriano Souto-Rey ${ }^{4}$ V Vanesa Balboa-Barreiro ${ }^{3}$ \\ ${ }^{1}$ Departamento de Ciencias de la Salud, Facultad de Enfermería y Podología, Universidade da Coruña (UDC); ${ }^{2}$ Grado en Podología, UDC; ${ }^{3}$ Grupo \\ de Investigación de Epidemiología Clínica, Departamento de Ciencias de la Salud, Facultad de Enfermería y Podología, UDC; ${ }^{4}$ Servicio de Cirugía \\ Ortopédica y Traumatología, Complejo Hospitalario Universitario de Ferrol (Área Sanitaria de Ferrol, España), Departamento de Ciencias de la \\ Salud, Facultad de Enfermería y Podología, UDC; Ferrol, A Coruña, España
}

\section{Resumen}

Objetivo: Conocer la prevalencia de onicocriptosis y de onicomicosis confirmada por cultivo en personas que acuden a una Clínica Universitaria de Podología, y describir sus hábitos podológicos. Método: Estudio transversal de prevalencia. El estudio fue llevado a cabo en la Clínica Universitaria de Podología de la Universidade da Coruña (Ferrol, España). Revisión de 1082 historias clínicas, de las cuales se seleccionaron 170 correspondientes a personas que presentaban onicocriptosis diagnosticada clínicamente y presencia de onicomicosis confirmada mediante cultivo. Se realizó un cuestionario de hábitos podológicos. Resultados: Edad media de la muestra $(n=1082): 47.9 \pm 22.1$ años. Prevalencia de onicocriptosis: $15.7 \%(n=170)$, con una edad media de $54.1 \pm 20.1$ años y con una afectación significativamente superior en mujeres y en menores de 65 años. La prevalencia de onicomicosis fue del 17.6\% $(n=30)$, con una edad media de $59.5 \pm 20.5$ años, mayor afectación en mujeres y en mayores de 65 años. En cuanto al cuestionario de hábitos, el calzado más empleado fue el cerrado, acordonado y de tacón plano. La mayoría de los sujetos acudían al podólogo por primera vez, caminaban aproximadamente 1 hora y su actividad diaria la desarrollaban de pie con desplazamientos cortos. Conclusiones: Se ha encontrado una alta prevalencia de onicocriptosis y de onicomicosis en personas demandantes de servicios de podología. Por su parte, utilizar un tipo de tacón plano o bajo y quién realice el cuidado de las uñas de los pies se muestran como variables predictoras de onicomicosis.

PALABRAS CLAVE: Cuidado del pie. Enfermedad del pie. Hábitos podológicos. Onicocriptosis. Onicomicosis. Patología ungueal.

\begin{abstract}
Objective: To determine the prevalence of onychocryptosis and onychomycosis confirmed by culture in subjects attending a Podiatric University Hospital and to describe their podiatric habits. Method: Cross-sectional study of prevalence. The study was carried out at the Clinic University of Podology of A Coruna University (Ferrol, Spain). Review of 1082 clinical histories, of which 170 were selected, persons who had onychocryptosis diagnosed clinically. The presence of onychomycosis confirmed by cultivation, presence of onychocryptosis through clinical diagnosis and a questionnaire was handed out of podologic habits. Results: Mean age of the total sample $(n=1082)$ was $47.9 \pm 22.1$ years. Prevalence of onychocryptosis was $15.7 \%(n=170)$ with a mean age of $54.1 \pm 20.1$ years and with a significantly higher affectation in women and people under 65. Prevalence of onychomycosis was $17.6 \%(n=30)$, mean age $59.5 \pm 20.5$ years with greater involvement in women and people over 65 years. With regard to the questionnaire of habits, the footwear more employee was the closed, cordoned off and heel flat. The majority of the subject came to a podiatrist for the first time; walked approximately 1 hour and their daily activity made of foot with short displacements. Conclusions: A high prevalence of onychocryptosis and onychomycosis in people attending Podiatric services has been found. For its part using a type of flat or low heel and who does the care of toenails are shown as predictive variables of onychomycosis.
\end{abstract}

KEY WORDS: Foot care. Foot disease. Podiatric habits. Onychocryptosis. Onychomycosis. Nail pathology.

Correspondencia:

Cristina González-Martín

Campus Universitario de Esteiro, $\mathrm{s} / \mathrm{n}$

Fecha de recepción en versión modificada: 24-11-2016

C.P. 15403, Ferrol, A Coruña, España

Fecha de aceptación: 04-01-2017

E-mail: cristina.gmartin@udc.es

DOI://dx.doi.org/10.24875/GMM.17003023

Gac Med Mex. 2017;153:810-817

Contents available at PubMed

www.gacetamedicademexico.com 


\section{Introducción}

Las afecciones podológicas constituyen un problema altamente prevalente en la población, especialmente en los mayores de 65 años ${ }^{1}$. En este sentido, Munro y Steele 2 refieren que el $70 \%$ de los mayores de 65 años padece algún tipo de problema en los pies. Recientemente, en España se ha observado que los dedos en garra (69.7\%) y el hallux valgus (38\%) constituyen las alteraciones podológicas más frecuentes en los mayores de 40 años $^{3}$. De manera secundaria, ambas afecciones pueden actuar como factores etiológicos de onicocriptosis (ONC), patología que a menudo se acompaña de dolor, inflamación e infección que, de cronificarse, provoca la aparición de tejido de granulación que cubre el surco ungueal y complicaciones mayores como la osteomielitis ${ }^{4-6}$. La epidemiología de la ONC es variable, y aunque no existe unanimidad en cuanto a qué sexo se ve más afectado, la mayoría de los autores están de acuerdo en que se manifiesta con mayor frecuencia en adolescentes y adultos jóvenes en la segunda y la tercera décadas de la vida. Al mismo tiempo, se considera más prevalente en el primer dedo del pie, siendo el canal peroneal el más afectado, con una relación 2:1 respecto al tibial ${ }^{7-10}$. Por su parte, la incidencia de onicomicosis (ONM) ha experimentado un aumento en los últimos años debido principalmente al envejecimiento de la población. Supone entre el 18 y el $40 \%$ de los trastornos ungueales, y su prevalencia se sitúa entre el 2 y el 18\% de la población mundial ${ }^{7,11-13}$. Ambas patologías (ONC y ONM) constituyen alteraciones ungueales muy comunes en la consulta podológica diaria, observándose que a partir de la tercera década de la vida la ONC se asocia en mayor medida a infecciones onicomicóticas, aunque también puede aparecer como complicación de la terapia antimicótica oral ${ }^{4,7,14-16}$.

\section{Método}

Se realizó un estudio transversal en la Clínica Universitaria de Podología de la Universidade da Coruña (Ferrol, España) mediante la revisión del historial clínico de las personas que acudieron a consulta por primera vez y por cualquier motivo entre septiembre de 2012 y septiembre de 2014. Durante ese periodo se estimó que acudirían a consulta alrededor de 1000 personas, tamaño muestral que para una seguridad del $95 \%$ y una precisión del $\pm 3 \%$ permite conocer las características presentadas por estas personas en las patologías a estudiar (ONC y ONM). Los datos fueron registrados por un solo clínico, siendo incluidos todos aquellos sujetos que dieron de manera libre y voluntaria su consentimiento escrito. Se excluyeron personas con amputación de alguna de sus extremidades inferiores, personas con sospecha de ONM pero que presentaron cultivo negativo, y todas aquellas que no dieron consentimiento escrito, siendo la muestra final de 1082 historias clínicas revisadas. De cada historial clínico se recogieron variables sociodemográficas (edad y sexo) y clínicas (presencia de ONC). Además, de los 1082 historiales revisados se seleccionaron aquellas personas que presentaron ONC diagnosticada clínicamente para recoger la información relativa al cuestionario de hábitos podológicos que por protocolo se cumplimenta durante la primera visita (Fig. 1) y para determinar la presencia de ONM confirmada por cultivo.

El estudio contó con la aprobación del Comité de Ética de la Investigación del Servicio Gallego de Salud (Xunta de Galicia, España) con código de registro 2015/113. En todo momento se siguieron las normas éticas recogidas en los reglamentos internacionales para la evaluación ética de los estudios epidemiológicos y de la investigación biomédica en sujetos humanos, y se aseguró el anonimato del paciente respetando la Ley de Protección de Datos de Carácter Personal (Ley Orgánica 15/1999 de 13 de diciembre), la Ley 41/2002 (básica reguladora de la autonomía del paciente y de derechos y obligaciones en materia de información y documentación clínica), la Ley 3/2001 (reguladora del consentimiento informado y de la historia clínica de los pacientes) y la Ley 3/2005 de modificación de la Ley 3/2001.

La información se analizó con el paquete estadístico IBM SPSS ${ }^{\circledR}$ (versión 20.0) para Windows ${ }^{\circledR}$ y se realizó un análisis descriptivo de las variables incluidas en el estudio. Las variables cuantitativas se expresaron como media, y las variables cualitativas, como valor absoluto (n) y porcentaje. Para comparar medias de variables cuantitativas en relación con variables cualitativas dicotómicas se realizó la prueba $t$ de Student 0 el test de Mann-Whitney, según procediera tras comprobación de la normalidad de las variables cuantitativas con el test de Kolmogorov-Smirnov. La asociación de variables cualitativas se estimó por medio del estadístico ji al cuadrado y a su vez se estimó el riesgo de presencia del evento calculando la odds ratio y su intervalo de confianza del $95 \%$. 

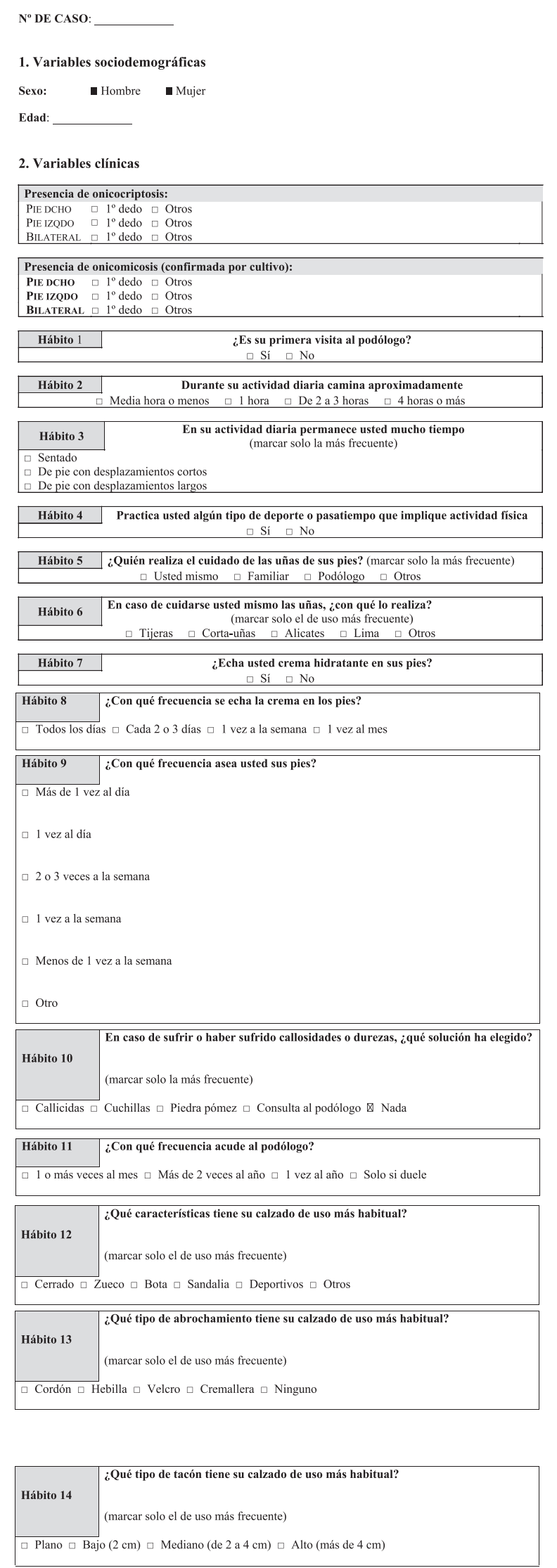

Figura 1. Cuestionario de hábitos podológicos.
Tabla 1. Descripción de los pacientes según edad, sexo y número de localizaciones de onicocriptosis y onicomicosis

\begin{tabular}{lccc}
\hline & $\begin{array}{c}\text { Onicocriptosis } \\
(\mathbf{n}=\mathbf{1 7 0})\end{array}$ & & $\begin{array}{c}\text { Onicomicosis } \\
(\mathbf{n}=\mathbf{3 0})\end{array}$ \\
\cline { 2 - 2 } & Media $\pm \mathbf{~ D T}$ & & Media $\pm \mathbf{~ D T}$ \\
\hline Edad & $54,09 \pm 20,09$ & & $58,57 \pm 19,86$ \\
Número de localizaciones & $1.56 \pm 0.62$ & & $1.2 \pm 0.41$ \\
\hline & $\mathbf{n}(\%)$ & $\mathbf{n}(\%)$ \\
\hline Sexo & & \\
Hombre & $49(28.8)$ & $11(36.7)$ \\
Mujer & $121(71.2)$ & $19(63.3)$ \\
Localizaciones & & \\
Pie derecho & & \\
Primer dedo & & $16(9.4)$ \\
Otros dedos & $127(74.7)$ & \\
Pie izquierdo & $8(4.7)$ & \\
Primer dedo & $122(71.8)$ & $17(10)$ \\
Otros dedos & $9(5.3)$ & $1(3.3)$ \\
Ambos pies primer dedo & $83(48.8)$ & $5(16.7)$ \\
\hline DT: desviación tínica & &
\end{tabular}

\section{Resultados}

Las características generales de la muestra estudiada se presentan en la tabla 1 , en la que se objetiva que la media de edad en los 170 pacientes con ONC es de 54.09 años $( \pm 20.09)$ y que predomina el sexo femenino $(71.2 \%$ vs. $28.8 \%)$. El número medio de localizaciones en dichos pacientes es de $1.56( \pm 0.62)$, predominando las localizaciones en el primer dedo del pie, tanto en el izquierdo como en el derecho ( $71.8 \%$ vs. $74.7 \%$ ). El $48.8 \%$ de las personas presentan localizaciones de dicha patología en ambos pies. De las 170 personas con ONC, 30 (17.6\%) presentan ONM, con una media de edad de 58.57 años $( \pm 19.86)$ y predominio también del sexo femenino $(63.3 \%$ vs. $36.7 \%)$.

El número medio de localizaciones de ONM en estos pacientes es de $1.2( \pm 0.41)$, predominando igualmente las localizaciones en el primer dedo tanto del pie izquierdo como del pie derecho $(9.4 \%$ vs. $10 \%)$.

Los hábitos podológicos en función de la patología (ONC u ONM) se muestran en la tabla 2. De las personas que presentan ONC, un $71.3 \%$ se cortan las uñas ellos mismos, utilizando cortaúñas un $37.7 \%$. En caso de sufrir callosidades o durezas, el método más utilizado es la piedra pómez (29.5\%). El calzado más habitual es el cerrado (58.7\%), y la forma de abrochamiento más común es el cordón (58.7\%), siendo el tacón plano el más empleado (44.7\%). En cuanto a 
Tabla 2. Descripción de los pacientes según sus hábitos en función del tipo de patología

\begin{tabular}{|c|c|c|}
\hline \multirow[t]{2}{*}{ Hábitos } & $\begin{array}{l}\text { Onicocriptosis } \\
\quad(n=170)\end{array}$ & $\begin{array}{l}\text { Onicomicosis } \\
\quad(n=30)\end{array}$ \\
\hline & $\mathrm{n}(\%)$ & n (\%) \\
\hline \multicolumn{3}{|l|}{1} \\
\hline No & $68(47.2)$ & $12(50.0)$ \\
\hline Sí & $76(52.8)$ & $12(50.0)$ \\
\hline \multicolumn{3}{|l|}{2} \\
\hline Menos de media hora & $51(30.0)$ & $11(44.0)$ \\
\hline 1 hora & $58(34.1)$ & $8(32.0)$ \\
\hline Entre 2 y 3 horas & $20(11.8)$ & $3(12.0)$ \\
\hline 4 o más horas & $12(7.1)$ & \\
\hline 3 & & $3(12.0)$ \\
\hline Sentado & $43(25.3)$ & $8(32.0)$ \\
\hline De pie/desplazamientos cortos & $76(44.7)$ & $15(60.0)$ \\
\hline De pie/desplazamientos largos & $27(15.9)$ & $2(8.0)$ \\
\hline \multicolumn{3}{|l|}{4} \\
\hline No & $34(36.9)$ & $5(35.7)$ \\
\hline Sí & $58(63.1)$ & $9(64.3)$ \\
\hline \multicolumn{3}{|l|}{5} \\
\hline Usted mismo & $107(71.3)$ & $21(80.8)$ \\
\hline Un familiar & $20(13.3)$ & 0 \\
\hline El podólogo & $15(10.0)$ & $5(19.2)$ \\
\hline Otros & $11(7.3)$ & $2(7.7)$ \\
\hline \multicolumn{3}{|l|}{ 5-rec } \\
\hline Usted mismo o profesional & $121(80.7)$ & $25(96.2)$ \\
\hline No profesional & $29(19.3)$ & $1(3.8)$ \\
\hline \multicolumn{3}{|l|}{6} \\
\hline Tijeras & $43(28.5)$ & $6(23.1)$ \\
\hline Cortaúñas & $57(37.7)$ & $9(34.6)$ \\
\hline Alicates & $38(25.2)$ & $8(30.8)$ \\
\hline Lima & $21(13.9)$ & $2(7.7)$ \\
\hline \multicolumn{3}{|l|}{7} \\
\hline No & $82(55.8)$ & $17(70.8)$ \\
\hline Sí & $65(44.2)$ & $7(29.2)$ \\
\hline \multicolumn{3}{|l|}{8} \\
\hline A diario & $22(31.4)$ & $3(42.9)$ \\
\hline Cada 2-3 días & $25(35.7)$ & $1(14.3)$ \\
\hline 1 vez a la semana & $17(24.3)$ & $1(14.3)$ \\
\hline 1 vez al mes & $6(8.6)$ & $2(28.6)$ \\
\hline \multicolumn{2}{|l|}{9} & $2(8.7)$ \\
\hline Más de 1 vez al día & $29(20.3)$ & $15(65.2)$ \\
\hline 1 vez al día & $78(54.5)$ & $4(17.4)$ \\
\hline $2-3$ veces por semana & $31(21.7)$ & $2(8.7)$ \\
\hline 1 vez por semana & $4(2.8)$ & $\begin{array}{c}210.11 \\
0\end{array}$ \\
\hline Otros & $1(0.7)$ & \\
\hline \multicolumn{3}{|l|}{10} \\
\hline Callicidas & $11(9.0)$ & 0 \\
\hline Cuchillas & $18(14.8)$ & $1(5.0)$ \\
\hline Piedra pómez & $36(29.5)$ & $5(25.0)$ \\
\hline Consulta podólogo & $31(25.4)$ & $5(25.0)$ \\
\hline Nada & $30(24.6)$ & $7(35.0)$ \\
\hline \multicolumn{3}{|l|}{11} \\
\hline 1 vez al mes & $11(19.3)$ & $1(3.3)$ \\
\hline
\end{tabular}

(Continúa)
Tabla 2. Descripción de los pacientes según sus hábitos en función del tipo de patología (Continuación)

\begin{tabular}{lcc}
\hline Hábitos & $\begin{array}{c}\text { Onicocriptosis } \\
(\mathbf{n}=\mathbf{1 7 0})\end{array}$ & $\begin{array}{c}\text { Onicomicosis } \\
(\mathbf{n}=\mathbf{3 0})\end{array}$ \\
\cline { 2 - 3 } & $\mathbf{n}(\%)$ & $\mathbf{n}(\%)$ \\
\hline Más de 2 veces al año & $9(15.8)$ & $4(40.0)$ \\
1 vez al año & $5(8.8)$ & $1(10.0)$ \\
Solo si duele & $32(56.1)$ & $4(40.0)$ \\
12 & & \\
Cerrado & $88(58.7)$ & $16(59.3)$ \\
Zueco & $20(13.3)$ & $2(7.4)$ \\
Bota & $34(22.7)$ & $5(18.5)$ \\
Sandalia & $29(19.3)$ & $3(11.1)$ \\
Deportivos & $53(35.3)$ & $12(44.4)$ \\
Otros & $9(6.0)$ & 0 \\
12-rec & & \\
Cerrado & $121(80.7)$ & $24(88.9)$ \\
Abierto & $29(19.3)$ & $3(11.1)$ \\
13 & & $14(58.3)$ \\
Cordón & & \\
Hebilla & & $1(4.2)$ \\
Velcro & $7(5.7)$ & $4(16.7)$ \\
Cremallera & $29(21.0)$ & $2(8.3)$ \\
Ninguna 14-rec & $22(15.9)$ & $4(16.7)$ \\
14-rec & $37(26.8)$ & $19(95.0)$ \\
Plano-bajo & & \\
Medio-alto & $96(73.3)$ & \\
\hline
\end{tabular}

Tabla 3. Análisis bivariado para predecir onicomicosis según las variables edad y número de localizaciones

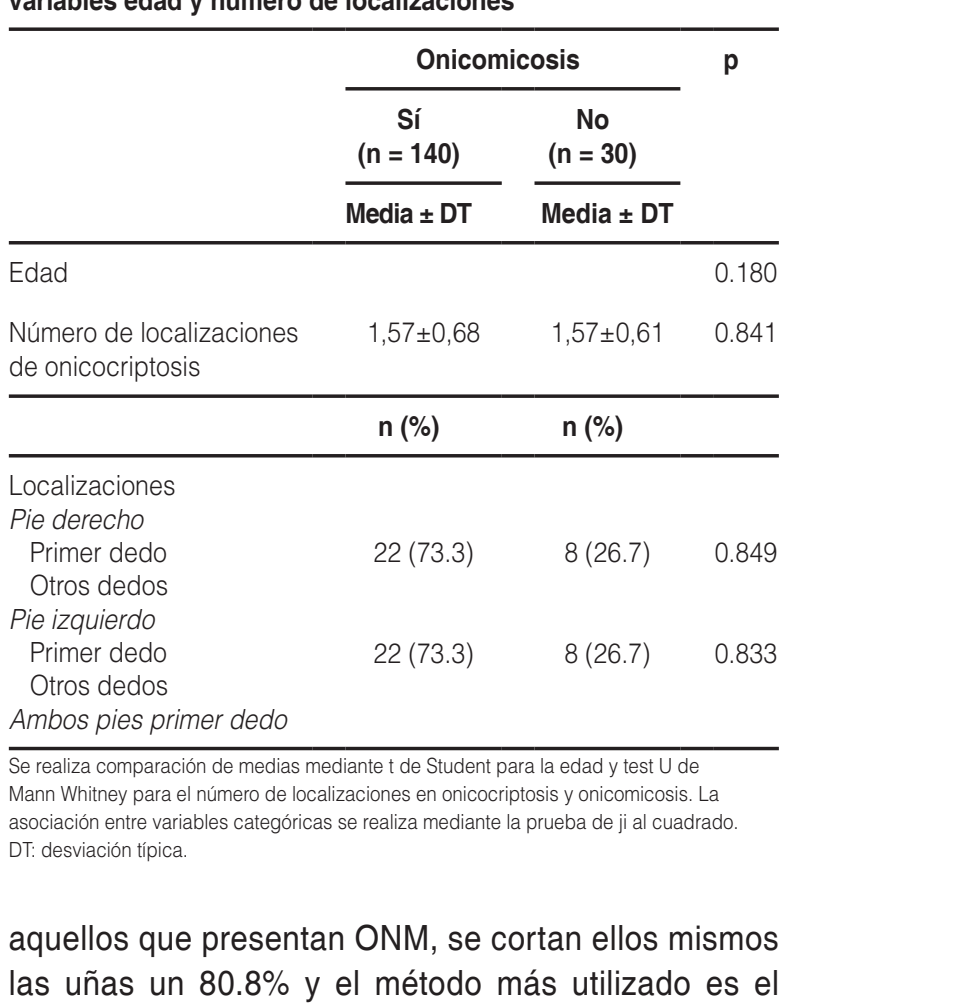
cortaúñas (34.6\%). Si sufren callosidades o durezas, un $35 \%$ no hace nada para solucionarlo. El calzado 
Tabla 4. Análisis bivariado para predecir onicomicosis según los hábitos de los pacientes

\begin{tabular}{|c|c|c|c|}
\hline \multirow[t]{3}{*}{ Hábitos } & \multicolumn{2}{|c|}{ Onicomicosis } & \multirow[t]{3}{*}{$p$} \\
\hline & \multirow{2}{*}{$\begin{array}{c}\begin{array}{c}\text { Sí } \\
(n=30)\end{array} \\
n(\%)\end{array}$} & \multirow{2}{*}{$\begin{array}{c}\begin{array}{c}\text { No } \\
(n=140)\end{array} \\
n(\%)\end{array}$} & \\
\hline & & & \\
\hline \multicolumn{3}{|l|}{1} & 0.765 \\
\hline No & $12(50.0)$ & $56(46.7)$ & \\
\hline Sí & $12(50.0)$ & $64(53.3)$ & \\
\hline \multicolumn{3}{|l|}{2} & 0.644 \\
\hline Menos de media hora & $11(40.0)$ & $40(34.5)$ & 0.074 \\
\hline 1 hora & $8(32.0)$ & $50(43.1)$ & \\
\hline Entre 2 y 3 horas & $3(12.0)$ & $17(14.7)$ & \\
\hline 4 o más horas & $3(12.0)$ & $9(7.8)$ & \\
\hline \multicolumn{3}{|l|}{3} & 0.917 \\
\hline Sentado & $8(32.0)$ & $35(29.4)$ & \\
\hline De pie/desplazamientos cortos & $15(60.0)$ & $61(51.3)$ & \\
\hline De pie/desplazamientos largos & $2(8.0)$ & $25(21.0)$ & \\
\hline \multicolumn{3}{|l|}{4} & 0.028 \\
\hline No & $5(35.7)$ & $29(37.2)$ & $0.0<0$ \\
\hline Sí & $9(64.3)$ & $49(62.8)$ & \\
\hline \multicolumn{4}{|l|}{ 5-rec } \\
\hline Usted mismo o profesional & $25(96.2)$ & $96(77.4)$ & 0.028 \\
\hline No profesional & $1(3.8)$ & $28(22.6)$ & \\
\hline \multicolumn{4}{|l|}{6} \\
\hline Tijeras & $6(23.1)$ & $37(29.6)$ & \\
\hline Cortaúñas & $9(34.6)$ & $48(38.4)$ & \\
\hline Alicates & $8(30.8)$ & $30(24.0)$ & \\
\hline Lima & $2(7.7)$ & $19(15.2)$ & \\
\hline \multicolumn{3}{|l|}{7} & 0.105 \\
\hline No & $17(70.8)$ & 65 (52.8) & \\
\hline Sí & $7(29.2)$ & $58(47.2)$ & \\
\hline \multicolumn{4}{|l|}{8} \\
\hline A diario & $3(42.9)$ & $19(30.2)$ & \\
\hline Cada 2-3 días & $1(14.3)$ & $24(38.1)$ & \\
\hline 1 vez a la semana & $1(14.3)$ & $16(24.5)$ & \\
\hline 1 vez al mes & $2(28.6)$ & $4(6.3)$ & \\
\hline \multicolumn{4}{|l|}{9} \\
\hline Más de 1 vez al día & $2(8.7)$ & $27(22.5)$ & \\
\hline 1 vez al día & $15(65.2)$ & $63(52.5)$ & \\
\hline 2-3 veces por semana & $4(17.4)$ & $27(22.5)$ & \\
\hline 1 vez por semana & $2(8.7)$ & $2(1.7)$ & \\
\hline Menos de 1 vez por semana & $0(0.0)$ & $1(0.8)$ & \\
\hline Otros & & & \\
\hline \multicolumn{4}{|l|}{10} \\
\hline Callicidas & $0(0.0)$ & $11(10.8)$ & \\
\hline Cuchillas & $1(5.0)$ & $17(16.7)$ & \\
\hline Piedra pómez & $5(25.0)$ & $31(30.4)$ & \\
\hline Consulta podólogo & $5(25.0)$ & $26(25.5)$ & \\
\hline Nada & $7(35.0)$ & $23(22.5)$ & \\
\hline \multicolumn{4}{|l|}{11} \\
\hline 1 vez al mes & $1(10.0)$ & $10(21.3)$ & \\
\hline Más de 2 veces al año & $4(40.0)$ & $5(10.6)$ & \\
\hline 1 vez al año & $1(10.0)$ & $4(8.5)$ & \\
\hline Solo si duele & $4(40.0)$ & $28(59.6)$ & \\
\hline
\end{tabular}

(Continúa)
Tabla 4. Análisis bivariado para predecir onicomicosis según los hábitos de los pacientes (Continuación)

\begin{tabular}{|c|c|c|c|}
\hline \multirow[t]{3}{*}{ Hábitos } & \multicolumn{2}{|c|}{ Onicomicosis } & \multirow[t]{3}{*}{$\mathrm{p}$} \\
\hline & $\begin{array}{c}\text { Sí } \\
(n=30)\end{array}$ & $\begin{array}{c}\text { No } \\
(n=140)\end{array}$ & \\
\hline & n (\%) & n (\%) & \\
\hline 12 & & & 0.232 \\
\hline Cerrado & $24(88.9)$ & $97(78.9)$ & \\
\hline Abierto & $3(11.1)$ & $26(21.1)$ & \\
\hline \multicolumn{4}{|l|}{13} \\
\hline Cordón & $14(58.3)$ & $67(58.8)$ & \\
\hline Hebilla & $1(4.2)$ & $6(5.3)$ & \\
\hline Velcro & $4(16.7)$ & $25(21.9)$ & \\
\hline Cremallera & $2(8.3)$ & $20(17.5)$ & \\
\hline Ninguna & $4(16.7)$ & $33(28.9)$ & \\
\hline 14 & & & 0.017 \\
\hline Plano-bajo & $19(95.0)$ & 77 (69.4) & \\
\hline Mediano-alto & $1(5.0)$ & $34(30.6)$ & \\
\hline
\end{tabular}

más utilizado es el cerrado (59.3\%), con abrochamiento mediante cordón (58.3\%) y con tacón plano $(47.6 \%)$.

Realizando un análisis bivariado para determinar si la edad, la localización y el número de localizaciones predicen la presencia de ONM, objetivamos que ninguna de las variables citadas tiene efecto independiente para predecir dicha patología (Tabla 3).

Para describir qué variables pueden ser predictoras de ONM se realizó un análisis bivariado según los hábitos de las personas estudiadas (Tabla 4). Además, se realizó un análisis bivariado de regresión logística para precisar si los hábitos podológicos 1 («¿Es su primera visita al podólogo?»), 2 («¿Durante su actividad diaria cuánto camina aproximadamente?»), 4 («¿Practica usted algún tipo de deporte o pasatiempo que implique actividad física?»), 5 («¿Quién realiza el cuidado de las uñas de sus pies?»), 7 («¿Pone usted crema hidratante en sus pies?»), 12 («¿Qué características tiene su calzado de uso más habitual?») y 14 («¿Qué tipo de tacón tiene su calzado de uso más habitual?»)) predicen la presencia de ONM. Observamos que los hábitos 5 y 14 presentan un efecto independiente para predecir la ocurrencia de dicha patología en el sentido de que el hecho de acudir a un profesional (podólogo) se relaciona con una mayor probabilidad de sufrir ONM. Esta relación puede deberse a que un motivo de consulta para acudir al podólogo es presentar alteraciones en las uñas, de la misma forma que aquellas personas que no acuden a un especialista es menos probable que puedan ser diagnosticadas de ONM. Con respecto al hábito 14, se 
observa que las personas que utilizan tacón plano o bajo presentan mayor probabilidad de sufrir ONM. Esto puede deberse a que aquellos que usan tacón plano o bajo son personas que utilizan zapatos cerrados (74.7\%), y entre ellos calzado deportivo (86.4\%). En el límite de la significación estadística se encuentra el hábito 7 , en el sentido de que cumplir este hábito se asocia a una mayor probabilidad de padecer ONM. EI resto de los hábitos estudiados en el análisis bivariado no tienen efecto independiente para la predicción de la presencia de ONM.

En el análisis bivariado para determinar una posible asociación entre la localización de ONC y el tipo de calzado encontramos en el límite de la significación estadística la asociación entre el tipo de calzado y la localización de ONC en el pie izquierdo (primer dedo) $(p=0.081)$, de manera que el hecho de usar calzado cerrado se asocia con la presencia de ONC en el primer dedo de este pie (Tabla 5).

Por último, se realizó también un análisis bivariado para determinar la asociación entre la localización de ONM y el tipo de calzado y la altura del tacón (Tabla 6). Objetivamos que utilizar tacón plano-bajo está asociado a la presencia de ONM en el primer dedo del pie izquierdo, no mostrándose asociación estadísticamente significativa con el resto de las localizaciones de ONM.

\section{Discusión}

Las onicopatías representan aproximadamente el $10 \%$ de todas las enfermedades de la piel ${ }^{11,17}$, y de ellas la ONM supone en torno al $50 \%{ }^{11}$. Son diversos los autores que han tratado de establecer la prevalencia de la ONC. Lloyd y Brill ${ }^{18}$ afirman que afecta al $20 \%$ de las personas con alguna patología del pie, mientras que $\mathrm{Krausz}^{19}$ señala que el $61 \%$ de las personas presenta algún tipo de patología ungueal, destacando sobre todas ellas la ONC. En la muestra estudiada se ha hallado una prevalencia de ONC del $15.7 \%$, con una mayor afectación en el sexo femenino y en menores de 65 años. Esta cifra es muy similar a la publicada en población gallega mayor de 65 $a_{n}{ }^{20}{ }^{20}$. No existe unanimidad en cuanto a qué sexo se ve más afectado por la ONC. Mientras que García y Fernánde $z^{4}$ objetivan una mayor frecuencia en el sexo femenino, otros autores señalan que es más frecuente en el sexo masculino ${ }^{8,21}$. Por su parte, la ONM está considerada como la principal causa de afección ungueal en los países desarrollados. En los últimos años ha experimentado un incremento en su prevalencia, generando gastos considerables al sistema de salud ${ }^{13}$. En nuestro estudio, la prevalencia de ONM en sujetos con ONC ascendió al 17.6\%, cifra concordante con lo publicado anteriormente ${ }^{20}$, y se localizó con más frecuencia en el primer dedo del $\mathrm{pie}^{22}$. Este resultado avalaría la tesis de que la prevalencia de ONM en sujetos con ONC no es superior a la de aquellos sin diagnóstico de ONC, ya que la cifra obtenida se encuentra dentro del rango descrito para la población general'12.

Cabe señalar que no hemos encontrado estudios en población española que hayan determinado la prevalencia de ONM en personas con ONC. Los datos más similares los hallamos en un estudio en el que, mediante revisión bibliográfica, se analizó la incidencia de ONC en pacientes tratados con antifúngicos orales $^{23}$. Otros estudios llevados a cabo en América del Norte y México han observado una incidencia de ONC de entre el 18 y el $37 \%$ tras tratamiento antimicótico por vía oral ${ }^{14,15}$.

En relación con los hábitos podológicos, un estudio en mayores de 65 años observó que el $55 \%$ de ellos aseaba sus pies a diario, menos de la mitad usaba crema hidratante, la mayoría realizaba un autocuidado de las uñas y solo un $28.9 \%$ recurrió a un podólogo ante un problema de hiperqueratosis o helomas ${ }^{24}$. Estas cifras son muy similares a las del presente estudio (54.5, 44.2, 71.4 y $26.1 \%$, respectivamente), si bien en nuestro caso la mayoría de los sujetos con ONC tenían menos de 65 años. En otro estudio llevado a cabo entre estudiantes de la Universidad Complutense de Madrid (España), la totalidad de los participantes afirmó haber acudido en alguna ocasión al podólogo y el $72 \%$ de ellos realizaban una hidratación diaria de los pies $^{25}$.

Se han publicado pautas para una correcta higiene y cuidado ungueal y del pie en general. En este sentido, es recomendable un lavado diario de los pies y la posterior aplicación de crema hidratante ${ }^{26,27}$. En ocasiones se plantea el uso de una piedra pómez para la eliminación de durezas, una lima de cartón o esmeril para un correcto corte de las uñas, o la visita regular al podólogo. En términos generales, son muy escasos los estudios que analizan los hábitos de higiene y cuidado del pie en la población general; sin embargo, sí son abundantes en población diabética ${ }^{28}$.

En cuanto al calzado, sabemos que un calzado adecuado y en buen estado puede ayudar a mantener el equilibrio, evitar el dolor y contribuir al bienestar general y a la independencia individual para las actividades de 
Tabla 5. Asociación entre las localizaciones de onicocriptosis y el tipo de calzado (hábito 12) y la altura del tacón (hábito 14)

\begin{tabular}{llcc}
\hline Hábito 12 & \multicolumn{2}{c}{$\begin{array}{c}\text { Localización en pie } \\
\text { izquierdo, primer dedo }\end{array}$} & $\mathbf{p}$ \\
\cline { 2 - 3 } & \multicolumn{1}{c}{ Sí } & No & \\
\hline Cerrado & $85(77,3)$ & $36(90.0)$ & 0.081 \\
Abierto & $25(22.7)$ & $4(10.0)$ & \\
\hline
\end{tabular}

Localización en pie izquierdo, otros dedos

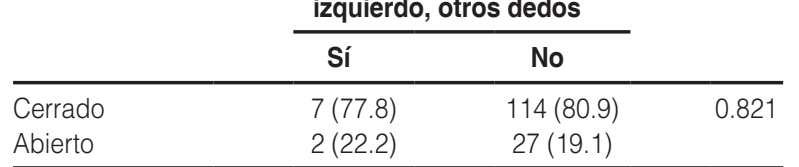

\begin{tabular}{lccc}
\hline & \multicolumn{2}{c}{$\begin{array}{l}\text { Localización en pie } \\
\text { derecho, primer dedo }\end{array}$} & \\
\cline { 2 - 3 } & \multicolumn{1}{c}{ Sí } & No & \\
\hline Cerrado & $92(81.4)$ & $29(78.4)$ & 0.685 \\
Abierto & $21(18.6)$ & $8(21.6)$ & \\
\hline
\end{tabular}

\begin{tabular}{|c|c|c|c|}
\hline & \multicolumn{2}{|c|}{$\begin{array}{l}\text { Localización en pie } \\
\text { derecho, otros dedos }\end{array}$} & \\
\hline & Sí & No & \\
\hline Cerrado & $3(37.5)$ & $118(83.1)$ & 0.001 \\
\hline Abierto & $5(62.5)$ & $24(16.9)$ & \\
\hline
\end{tabular}

\begin{tabular}{|c|c|c|c|}
\hline & \multicolumn{2}{|c|}{$\begin{array}{l}\text { Localización bilateral, } \\
\text { primer dedo }\end{array}$} & \\
\hline & Sí & No & \\
\hline Cerrado & $58(76.3)$ & $63(85.1)$ & 0.171 \\
\hline Abierto & $18(23.7)$ & $11(14.9)$ & \\
\hline \multirow[t]{2}{*}{ Hábito 14} & \multicolumn{2}{|c|}{$\begin{array}{l}\text { Localización en pie } \\
\text { izquierdo, primer dedo }\end{array}$} & \\
\hline & Sí & No & \\
\hline Tacón plano-bajo & $70(71.4)$ & $26(78.8)$ & 0.409 \\
\hline Tacón medio-alto & $28(28.6)$ & $7(21.2)$ & \\
\hline
\end{tabular}

\begin{tabular}{|c|c|c|c|}
\hline & \multicolumn{2}{|c|}{$\begin{array}{l}\text { Localización en pie } \\
\text { izquierdo, otros dedos }\end{array}$} & \\
\hline & Sí & No & \\
\hline \multirow{4}{*}{$\begin{array}{l}\text { Tacón plano-bajo } \\
\text { Tacón medio-alto }\end{array}$} & $5(62.5)$ & $91(74.0)$ & 0.477 \\
\hline & $3(37.5)$ & $32(26.0)$ & \\
\hline & \multicolumn{2}{|c|}{$\begin{array}{l}\text { Localización en pie } \\
\text { derecho, primer dedo }\end{array}$} & $p$ \\
\hline & Sí & No & \\
\hline Tacón plano-bajo & $72(74.2)$ & $24(70.6)$ & 0.680 \\
\hline Tacón medio-alto & $25(25.8)$ & $10(29.4)$ & \\
\hline
\end{tabular}

\begin{tabular}{|c|c|c|c|}
\hline & \multicolumn{2}{|c|}{$\begin{array}{l}\text { Localización en pie } \\
\text { derecho, otros dedos }\end{array}$} & \\
\hline & Sí & No & \\
\hline $\begin{array}{l}\text { Tacón plano-bajo } \\
\text { Tacón medio-alto }\end{array}$ & $\begin{array}{l}6(85.7) \\
1(14.3)\end{array}$ & $\begin{array}{l}90(72.6) \\
34(27.4)\end{array}$ & 0.445 \\
\hline
\end{tabular}

\begin{tabular}{llll}
\hline & \multicolumn{2}{c}{$\begin{array}{c}\text { Localización bilateral, } \\
\text { primer dedo }\end{array}$} & \\
\cline { 2 - 3 } & Sí & No & \\
\hline Tacón plano-bajo & $46(69.7)$ & $50(76.9)$ & 0.350 \\
Tacón medio-alto & $20(30.3)$ & $15(23.1)$ & \\
\hline
\end{tabular}

Tabla 6. Asociación entre las localizaciones de onicomicosis y el tipo de calzado (hábito 12) y la altura del tacón (hábito 14)

\begin{tabular}{llcc}
\hline Hábito 12 & \multicolumn{2}{c}{$\begin{array}{c}\text { Localización en pie } \\
\text { izquierdo, primer dedo }\end{array}$} & $\mathbf{p}$ \\
\cline { 2 - 3 } & Sí & No & \\
\hline Cerrado & $14(93.3)$ & $107(79.3)$ & 0.190 \\
Abierto & $1(6.7)$ & $28(20.7)$ & \\
\hline
\end{tabular}

\begin{tabular}{lccc}
\hline & \multicolumn{3}{c}{$\begin{array}{c}\text { Localización en pie } \\
\text { izquierdo, otros dedos }\end{array}$} \\
\cline { 2 - 3 } & Sí & No & \\
\hline Cerrado & $1(100)$ & $120(80.5)$ & 1.000 \\
Abierto & 0 & $29(19.5)$ & \\
\hline
\end{tabular}

\begin{tabular}{llll}
\hline & \multicolumn{2}{c}{$\begin{array}{c}\text { Localización en pie } \\
\text { derecho, primer dedo }\end{array}$} & \\
\cline { 2 - 3 } & Sí & No & \\
\hline Cerrado & $11(78.6)$ & $110(80.9)$ & 0.835 \\
Abierto & $3(21.4)$ & $26(19.1)$ & \\
\hline
\end{tabular}

\begin{tabular}{lccc}
\hline & \multicolumn{3}{c}{$\begin{array}{l}\text { Localización en pie } \\
\text { derecho, otros dedos }\end{array}$} \\
\cline { 2 - 3 } & Sí & No & \\
\hline Cerrado & $2(100)$ & $119(80.4)$ & 1.000 \\
Abierto & 0 & $29(19.6)$ & \\
\hline
\end{tabular}

\begin{tabular}{llcl}
\hline & \multicolumn{2}{c}{$\begin{array}{c}\text { Localización bilateral, } \\
\text { primer dedo }\end{array}$} & \\
\cline { 2 - 3 } & Sí & No & \\
\hline Cerrado & $3(75.0)$ & $118(80.8)$ & 0.771 \\
Abierto & $1(25.0)$ & $28(19.2)$ & \\
\hline
\end{tabular}

\begin{tabular}{lccc}
\hline Hábito 14 & \multicolumn{2}{c}{ Localización en pie } \\
izquierdo, primer dedo & & \\
\cline { 2 - 3 } & Sí & No & \\
\hline Tacón plano-bajo & $11(100)$ & $85(70.8)$ & 0.036 \\
Tacón medio-alto & 0 & $35(29.2)$ & \\
\hline
\end{tabular}

\begin{tabular}{|c|c|c|c|}
\hline & \multicolumn{2}{|c|}{$\begin{array}{l}\text { Localización en pie } \\
\text { izquierdo, otros dedos }\end{array}$} & \\
\hline & Sí & No & \\
\hline \multirow{4}{*}{$\begin{array}{l}\text { Tacón plano-bajo } \\
\text { Tacón medio-alto }\end{array}$} & $1(100)$ & $95(73.1)$ & 1.000 \\
\hline & 0 & $35(26.9)$ & \\
\hline & \multicolumn{2}{|c|}{$\begin{array}{l}\text { Localización en pie } \\
\text { derecho, primer dedo }\end{array}$} & $p$ \\
\hline & Sí & No & \\
\hline Tacón plano-bajo & $9(90.0)$ & $87(71.9)$ & 0.214 \\
\hline Tacón medio-alto & $1(10.0)$ & $34(28.1)$ & \\
\hline
\end{tabular}

Localización en pie

\begin{tabular}{lccc} 
& \multicolumn{3}{c}{ derecho, otros dedos } \\
\cline { 2 - 3 } & Sí & No & \\
\hline Tacón plano-bajo & $1(100)$ & $95(73.1)$ & 1.000 \\
Tacón medio-alto & 0 & $35(26.9)$ & \\
\hline
\end{tabular}

\begin{tabular}{lccc}
\hline & \multicolumn{3}{c}{$\begin{array}{c}\text { Localización bilateral, } \\
\text { primer dedo }\end{array}$} \\
\cline { 2 - 3 } & Sí & No & \\
\hline Tacón plano-bajo & $2(100)$ & $94(72.9)$ & 1.000 \\
Tacón medio-alto & 0 & $35(27.1)$ & \\
\hline
\end{tabular}


la vida diaria; y que, por el contrario, el uso de un calzado inadecuado o defectuoso, que provoque microtraumatismos repetitivos o favorezca un ambiente húmedo, propicia el desarrollo de ONM y de otras lesiones como hiperqueratosis y helomas. En nuestro trabajo, el calzado más habitualmente utilizado fue el cerrado, coincidiendo con otros estudios llevados a cabo en población española mayor de 60 años $^{29}$. Sin embargo, no conocemos las características del calzado en cuanto a su horma o material de confección, pudiendo tratarse de zapatos estrechos, de longitud insuficiente o poco transpirables, en cuyo caso podrían ser considerados de riesgo para desarrollar las patologías en estudio. En población universitaria se ha observado que el calzado deportivo es el más empleado, con porcentajes incluso superiores al obtenido en nuestra población de estudio, probablemente debido a las diferencias en la edad media de los sujetos estudiados en uno y otro caso $^{30}$.

Estos resultados señalan a priori que la prevalencia de ONM en personas con ONC no es superior a la encontrada en sujetos no diagnosticados de ONC. Sin embargo, dada la escasez de estudios relativos a esta afección, creemos que es necesario seguir investigando para un mejor conocimiento de esta onicopatía tan común.

\section{Bibliografía}

1. Dunn JE, Link CL Felson DT. Prevalence of foot and ankle conditions in a multiethnic community sample of older adults. Am J Epidemiol. 2004;159:491-8.

2. Munro BJ, Steele JR. Foot-care awareness: a survey of persons aged 65 years and older. J Am Podiatr Med Assoc. 1998;88:242-8.

3. Pita Fernández S, González Martín C, Seoane Pillado T, et al. Podiatric medical abnormalities in a random population sample 40 years or older in Spain. J Am Podiatr Med Assoc. 2014:104:574-82.

4. García Carmona FJ, Fernández Morato D. Tratamiento quirúrgico de la onicocriptosis. Madrid: Aula Médica; 2003.

5. Novel Martí V. Cirugía ungueal. En: Izquierdo Cases JO, editor. Podología quirúrgica. Madrid: Elsevier; 2006. p. 41-53.
6. García Carmona FJ, Padín Galea JM, Fernández Morato D. Onicocriptosis crónica. Piel. 2005;20:53-5

7. Martínez Nova A. Podología: atlas de cirugía ungueal. Madrid: Panamericana; 2006.

8. Zavala Aguilar KZ, Gutiérrez Pineda F, Bozalongo de Aragón E. Manejo de la onicocriptosis en atención primaria. Caso clínico. Semergen. 2013;39:38-40.

9. Moreno Arroyo V, Granados Roldán MO, Tejera Vaquerizo AM. Onicocriptosis: un nuevo método terapéutico. Enferm Dermatol. 2009;3:36-7.

10. Fernández Arista G, Enríquez Merino J. Onicocriptosis: estudio comparativo del periodo posoperatorio de una matricectomía parcial lateral con el de una matricectomía parcial lateral con fenolización. Dermatol Rev Mex. 2006;50:87-93

11. Domingo Herranz H, Ribera Pibernat M. Onicopatías más frecuentes en atención primaria. Jano. 2005;69:49-59.

12. Larruskain Garmendia J, Idígoras Viedma P, Mendiola Arza J. Onicomicosis: diagnóstico y tratamiento. Información Terapéutica del Sistema Nacional de Salud. 2008;32:83-92.

13. Ballesté R, Mousqués N, Gezuele E. Onicomicosis: revisión del tema Rev Med Urug. 2003;19:93-106.

14. Connelley Jr LK, Dinehart SM, McDonald R. Onychocryptosis associated with the treatment of onychomycosis. J Am Podiatr Med Assoc. 1999;89:424-6.

15. Bonifaz A, Paredes V, Fierro L. Onychocryptosis as consequence of effective treatment of dermatophytic onychomycosis. J Eur Acad Dermaol. 2007:21:699-700.

16. Weaver TD, Jespersen DL. Multiple onychocryptosis following treatment of onychomycosis with oral terbinafine. Cutis. 2000;66:211-2.

17. Mosquera Fernández A, Bouza Vivero MC, Lluch Fruns J, et al. Abordaje conservador de la onicocriptosis leve. Enferm Dermatol. 2010;11:41-4.

18. Lloyd Davies RW, Brill GC. The aetiology and out-patient management of ingrowing toe-nails. Br J Surg. 1963;50:592-7.

19. Krausz CE. Nail survey (1942-1970). Br J Chirop. 1970:35:117.

20. Romero Soto M, Santalla Borreiros F, Gil Manso P, et al. Estudio de prevalencia de patologías podológicas y grado de dependencia en personas mayores de 65 años. Peu. 2010;30:104-11.

21. Murray WR, Bedi BS. The surgical management of ingrowing toenail. $\mathrm{Br}$ J Surg. 1975;62:409-12.

22. Nazar JR, Gerosa PE, Díaz OA. Onicomicosis: epidemiología, agentes causales y evaluación de los métodos diagnósticos de laboratorio. Rev Argent Microbiol. 2012;44:21-5

23. Corral Lizana N, Álvarez Arroyo J. Relación entre el uso de antimicóticos y el aumento en la incidencia de onicocriptosis. Reduca. 2012;4:11.

24. Santalla Borreiros F, Romero Soto M, Gil Manso P, et al. Hábitos de cuidados podológicos en personas mayores de 65 años. Peu. 2010;30:36-41.

25. Cañas López MV, Rodríguez Olvera D. ¿Qué llevas en los pies? Reduca. 2014;6:34-8

26. Gómez Ayala AE. Cuidados podológicos básicos. Farmacia Profesional. 2006;20:43-7.

27. Azcona Barbed L. Cuidados del pie. La salud en la base. Farmacia Profesional. 2004;18:58-62.

28. Fustero I. Cuidado de los pies. Offarm. 2007:26:66-72

29. Álvarez-Calderón Iglesias O, Alonso Tajes F, López López D, et al. Análisis del calzado en una población mayor de 60 años. Rev Int Cienc Podol. 2008;2:19-26.

30. Carlier Espeleta B, Santafé Martín C, Solano Alarcón R. Hábitos podológicos en una población universitaria. Reduca. 2011;3:5-6. 